\title{
Multiple Myeloma: An Uncommon Presentation of a Common Neurological Problem
}

\author{
Mansoor N Ali
}

Consultant Nephrologist, Renal Unit, Department of Renal Medicine, St. Luke's Hospital, Bradford Teaching Hospitals NHS Foundation Trust, BD5 0NA

Article Info

\section{Article Notes}

Received: July 04, 2020

Accepted: July 20, 2020

\section{${ }^{*}$ Correspondence:}

Dr Mansoor N Ali, Consultant Nephrologist, Renal Unit, Department of Renal Medicine, St. Luke's Hospital, Bradford Teaching Hospitals NHS Foundation Trust, BD5 ONA; Email: Mansoor.ali@nhs.net.

${ }^{\circ} 2020$ Ali MN. This article is distributed under the terms of the Creative Commons Attribution 4.0 International License.

\section{Keywords}

Multiple Myeloma

Radiculopathy

Spinal cord compression

Listening to patients

Communication.

\section{Introduction}

Multiple myeloma is a neoplastic proliferation of abnormal plasma cells; a clonal malignancy of terminally differentiated B lymphocytes. The clinical features of multiple myeloma is a result of either direct proliferation of the plasma cells causing extensive bony destructive lesions including pathological fractures or indirectly related to paraprotein production mainly monoclonal immunoglobulins resulting in renal damage e.g. proteinuria, renal impairment (acute kidney injury AKI), electrolyte disturbances and amyloid deposits. In addition to renal impairment; patients with multiple myeloma frequently develop complications related to their disease, including hypercalcemia, infection, and skeletal lesions which require specific treatment in addition to directed treatment at the malignant clone.

Multiple myeloma can also present with signs of spinal cord compression resulting in paraesthesia, paraplegia and bladder or bowel dysfunction and/or incontinence, which can be missed and may lead to delay in diagnosis and treatment.

Below is a case description where multiple admissions of a patient led to a delay in the diagnosis of spinal cord compression caused by multiple myeloma.

\section{Description}

\section{First presentation to emergency department: ED}

A 73 years old female patient presented to the emergency department following recurrent mechanical falls. Her past medical history included asthma on inhalers, atrial fibrillation on warfarin, bilateral knee replacements (last replacement 2 years prior to presentation). The falls were mainly at home and resulted in various bruises and bony aches and pains which responded to analgesia mainly Non-steroidal anti-inflammatory drugs (NSAIDs).

On admission, the patient had suffered pre-patellar lacerations which was treated in the emergency department with suturing of the wound and was subsequently discharged home on the same day. No laboratory tests were carried out during this admission.

\section{Second admission}

The patient further attended ED within a week with right knee pain, complaining of inability to walk. No formal neurological examination was carried out apart from documentation, that the patient's mobility was affected. The working diagnosis in ED was right knee haematoma due to raised international normalised ratio (INR of 5) as she had been on warfarin. The patient was transferred to 
the orthopaedic ward where she stayed as an inpatient for less than 2 weeks. She required knee washouts and multiple blood transfusions due to anaemia and was subsequently discharged with some physiotherapy support. She had a prolonged spell but the cause of inability to walk and anaemia was never fully investigated nor was any formal neurological examination carried out.

Initial labs showed haemoglobin $(\mathrm{Hb})$ of $70 \mathrm{~g} / \mathrm{L}$ (reference range female $115-160$ ), platelets of $180 \times 10^{9} / \mathrm{L}$ (reference range 150-400), INR 4.8, mean cell volume (MCV) of 80 $\mathrm{fL}$ (reference range 80-99) and mean cell haemoglobin concentration (MCHC) of $300 \mathrm{~g} / \mathrm{L}$ (reference range 300-370) . Renal functions showed a urea of $8.0 \mathrm{mmol} / \mathrm{L}$ (reference range 2.5-7.8) and creatinine $120 \mathrm{umol} / \mathrm{L}$ (reference range 49-90) from a baseline of $80 \mathrm{umol} / \mathrm{L}$ on the blood test done a year prior to admission and was thought to be pre renal AKI. The patient required multiple blood transfusions to keep $\mathrm{Hb}$ targets above $90 \mathrm{~g} / \mathrm{L}$. Her calcium level were mildly elevated at $2.81 \mathrm{mmol} / \mathrm{L}$ (reference range 2.20-2.60) but was down to having over the counter calcium supplements. Her alkaline phosphatase was within normal range of 120 IU/L (reference range 30-130).

There were no further investigations such as haematinics (iron, vitamin B12 or folate levels) or upper and lower gastrointestinal investigations; carried out to determine the cause of the anaemia during the second admission.

\section{Third admission}

The patient was re-admitted with recurrent falls four days after her last hospital discharge.

A brief neurology examination on admission was documented as grossly normal.

Further physiotherapy was arranged and plans made for discharge with outpatient physiotherapy. Patient was deemed medically fit but needed more physiotherapy and occupational therapy. Hb levels were $89 \mathrm{~g} / \mathrm{L}$, creatinine levels at $132 \mathrm{umol} / \mathrm{L}$, calcium of $2.86 \mathrm{mmol} / \mathrm{L}$.

During a weekend on call; the patient's husband asked the on call consultant to review his wife as he was very worried about general decline and lack of clinical progress. The husband asked for someone to listen to him and his wife as these repeated presentations with falls and difficulty in walking were unusual for his otherwise functionally independent wife.

\section{Listening to Patients and Relatives}

The on call consultant who had never met the patient before, listened to the husband and to their own instinct. Upon careful history taking and examination; the patient appeared to have had significant neurological signs of spinal cord compromise. There was weakness on both lower limbs 2/5 muscle power grade with up going plantar reflex in both lower limbs, significant hyperaesthesia and upon revisiting history; patient had been incontinent and had a recent catheterisation for an incomplete bladder emptying.

The examination findings were further discussed with the radiologist on call urgently and despite it being a weekend with no dedicated magnetic resonance (MR) specialist radiology on call, an urgent magnetic resonance imaging (MRI) was organised by the radiology department.

MRI showed significant spinal cord compression which was promptly reported by the on call radiologist (see Figure 1).

The patient was urgently referred to a neurosurgical centre for surgical decompression (see Figure 2). The ongoing anaemia also prompted the on call team to request haematinics and a myeloma screen which showed significant free light chain levels in the serum and presence of urine protein electrophoresis. Haematology consult

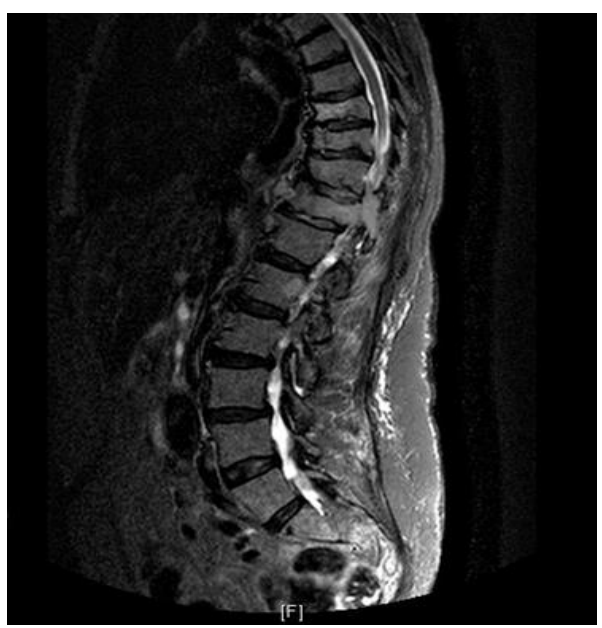

Figure 1: Sagittal T2 weighted image through the thoracolumbar spine showing abnormality in the T11 vertebra and right pedicle causing significant narrowing of the central spinal canal and contained spinal cord- appearances suggest acute spinal cord compromise.

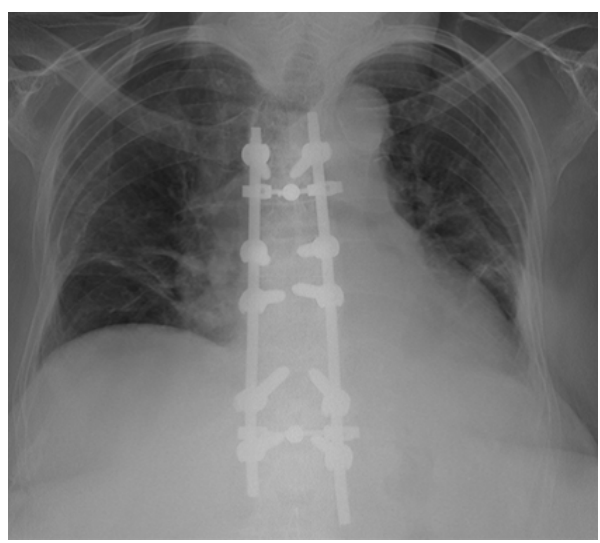

Figure 2: Post OP CXR after surgical decompression. 
was sought as an inpatient and a bone marrow biopsy was subsequently performed which confirmed myeloma and was treated with radiotherapy followed by chemotherapy.

The patient also had stage 1 Acute kidney injury (AKI); creatinine values of $140 \mathrm{umol} / \mathrm{L}$ from a baseline creatinine of around 70-80 umol/L, detected during her third admission which was initially put down to dehydration and possible urinary infection. Intravenous fluids were given but no improvement was seen in her kidney functions during the first and second episodes of her admission. The patient recovered her kidney functions during the course of the treatment for myeloma.

\section{Discussion}

Multiple myeloma represents $1-2 \%$ of all cancers and slightly more than $17 \%$ of all hematologic cancers ${ }^{1}$, 2. Multiple myeloma is a condition of malignant plasma cell proliferation derived from a single B-cell lineage ${ }^{3}$. Plasma cells produce monoclonal immunoglobulins, most commonly either immunoglobulin G (IgG) or immunoglobulin A (IgA) ${ }^{4}$. The vast majority of the patients with Multiple Myeloma will have a monoclonal M protein produced and secreted by the malignant plasma cells, which can be detected by serum free light chain (FLC) analysis and/or protein electrophoresis of the serum (SPEP) and urine ${ }^{5}$.

Radiculopathy, usually in the thoracic or lumbosacral area, is one of the most common neurologic complications of Multiple Myeloma ${ }^{6}$. It can result from compression of the nerves by plasmacytoma or rarely by the collapsed bone itself.

Spinal cord compression from multiple myeloma should be suspected in patients who present with back pain including symptoms of weakness or paraesthesia, bladder or bowel dysfunction and/or incontinence, including unexplained anaemia (requiring blood transfusions as in our patient's case). A normocytic, normochromic anaemia is present in $73 \%$ at diagnosis and $93 \%$ at some point during the course of the disease $\mathrm{e}^{5}$. This may or may not be associated with significant hypercalcemia.

Magnetic resonance imaging (MRI) or computed tomographic (CT) myelography of the entire spine should be performed immediately if this complication is suspected. Some of these patients may also have a prolonged history of analgesic use mainly Non-steroidal (NSAIDs), to alleviate back pain. The patient may have varying degrees of renal impairment. The rise in serum creatinine is seen in almost one half of the patients at diagnosis ( $>177$ umol/L in approximately $20 \%$ ), in some cases; renal insufficiency is the presenting manifestation of multiple myeloma ${ }^{5,7}$.

Prompt diagnosis and immediate treatment are of critical and paramount importance in the preservation of neurological function in patients with spinal cord compression.

The aims of treatment include management of pain, treating complications and managing them, supportive renal care including renal replacement therapy, and the preservation and/or improvement of neurologic functioning. The patient in the above case received surgical decompression, radiation therapy followed by CTD chemotherapy (cyclophosphamide, thalidomide, and dexamethasone). The patient remained under the care of haematology following neurosurgical intervention and was discharged home after intensive rehabilitation and physiotherapy along with the ongoing treatment and follow up for multiple myeloma.

It is important to bear in mind; in elderly patients who may well have underlying systemic illness or malignancy, they may present as being generally off colour, fatigued or have difficulty in mobility.

\section{Learning Points}

It is important to listen to patients and family when they mention to you that their "loved ones" are not feeling right.

Listening to patients and concentrating on their problems helps to:

- develop insight into their problems,

- increase efficiency and prompt delivery of care,

- achieve realistic goals in managing their conditions,

- understand expectations from both patients and relatives,

- enhance communication and avoids complaints,

- build trust and rapport, and

- improve quality and delivery of compassionate care.

Effective communication with both patients and relatives where they feel listened to have also formed part of the friends and family tests in the National Health Service (NHS) ${ }^{8}$.

General Medical Council (GMC) expects registered doctors to clearly demonstrate their duties to meet the standards as expected in the four Domains laid out in its guidance.

One of the domains is on "Communication, partnership and teamwork" which mentions ${ }^{9}$ in its paragraph No. 31.

You must listen to patients, take account of their views, and respond honestly to their questions.

The patient should feel that as health professionals:

- "We are here for them." 
- $\quad$ "There is no better way to understand how to help patients, than spending time listening to them."

\section{Conflict of Interest}

Author declare that there is no conflict of interest regarding the submission of article "Multiple Myeloma: An uncommon presentation of a common neurological problem"

\section{References}

1. Siegel RL, Miller KD, Jemal A. Cancer statistics 2020. CA Cancer J Clin 2020; 70:7.

2. SEER Stat Fact Sheets: Kidney and Renal Pelvis http://seer.cancer. gov/statfacts/html/kidrp.html

3. Enitza D, George MD, Richard Sadovsky MDMS. State University of New York Health Science Center, Brooklyn, New York Am Fam Physician. 1999 Apr 1; 59(7): 1885-1892.
4. Bataille R, Harousseau JL. Multiple myeloma. N Engl J Med. 1997; 336(23): 1657-1664. doi: 10.1056/NEJM199706053362307.

5. Kyle RA, Gertz MA, Witzig TE, et al. Review of 1027 patients with newly diagnosed multiple myeloma, Mayo Clin Proc. 2003; 78(1).

6. Ito H, Ishii K, Okada k, et al. Major Symptoms of Multiple Myeloma and Their Management--Neurologic Disorders Nihon Rinsho. 1995 Mar; 53(3): 684-9.

7. Winearls CG. Acute myeloma kidney. Kidney Int. 1995; 48: 1347.

8. McIntyre L. Implementing the Friends and Family Test. Nursing Times. 2014; 110(45): 12-14.

9. General Medical Council, Good Medical practice duties of a doctor https://www.gmc-uk.org/ethical-guidance/ethical-guidance-fordoctors/good-medical-practice 